\title{
Marrow Cytometry and Prognosis in Myeloma
}

\author{
Barthel Barlogie, Raymond Alexanian, Edmund A. Gehan, \\ Leslie Smallwood, Terry Smith, and Benjamin Drewinko, Departments of \\ Developmental Therapeutics, Medicine, Biomathematics, and Laboratory \\ Medicine, The University of Texas System Cancer Center, M. D. Anderson \\ Hospital and Tumor Institute, Houston, Texas 77030
}

A B S TRACT We have previously shown that flow cytometric analysis of acridine orange-stained bone marrow cells is useful for the objective enumeration and characterization of plasma cells from patients with myeloma, frequently exhibiting an abnormal DNA and an elevated RNA content. In this report on 77 previously untreated patients, we have investigated the biologic and prognostic implications of these quantitative tumor cell parameters. The degree of marrow involvement by tumor, both by microscopic and cytometric analysis, correlated with the clinically derived tumor mass stage. Examination of the product of relative tumor cell RNA content and marrow tumor infiltrate (as a measure of metabolic capacity for immunoglobulin production) in relationship to the myeloma protein concentration in the serum revealed differences in the efficiency of immunoglobulin production and/or catabolism. There was an inverse relationship between the degree of marrow tumor involvement and RNA index, suggesting a more aggressive behavior of myeloma in patients with a low tumor cell RNA content. Prognostically, high tumor cell RNA content identified patients with a high likelihood of response to both initial treatment (32 patients, $P$ $=0.004)$ and salvage therapy $(29$ patients, $P=0.01)$. Favorable factors for survival were low clinical tumor mass stage $(P=0.07)$ and low marrow tumor infiltrate as determined morphologically $(P=0.04)$ and cytometrically $(P=0.004)$. Thus, the direct examination of marrow cellular DNA and RNA content permitted assessment of tumor burden and was useful in the prediction of response and survival.

\section{INTRODUCTION}

The recognition of prognostic factors in patients with cancer has allowed earlier treatment of high risk pa-

Address reprint requests to Dr. Barthel Barlogie, The University of Texas System Cancer Center, Houston, TX 77030.

Received for publication 18 August 1982 and in revised form 27 May 1983. tients with new and potentially more effective agents. For most tumors, prognosis is adversely affected by increasing tumor burden. This also applies to myeloma where a staging system based on tumor load has been used to predict survival (1-3). Durie et al. (4) and Latreille et al. (5) have recently reported that pretreatment tumor cell proliferative activity and/or ploidy provide additional prognostic information.

Tumor cytoreduction by $\geq 75 \%$ has been observed in about one-half of patients treated with alkylating agent-prednisone combinations $(6,7)$. Unfortunately, no clinical or laboratory feature has consistently predicted response. Hofmann et al. (8) recently reported on the usefulness of tritiated thymidine grain count evaluation to predict both response and survival in myeloma patients, but the procedure is laborious and relies on microscopic identification of myeloma tumor cells after autoradiographic processing.

We have previously demonstrated that cytometry of DNA and RNA content is an objective means to quantitate tumor cells in the marrow of the majority of patients with myeloma, as well as in about one-half of patients with benign monoclonal gammopathy (5, $9,10)$. The proportion of cytometrically identifiable cells was similar to the proportion of morphologically recognizable plasma cells. In this report, we show that the determination of the proportion of both microscopically and cytometrically defined bone marrow tumor cells compares well with clinical estimates of tumor burden, hence permitting a more direct assessment of tumor mass stage by one single parameter. In addition, bone marrow tumor infiltrate and RNA content had prognostic implications both for response and for survival.

\section{METHODS}

We have studied 77 consecutive, previously untreated patients with overt multiple myeloma, who have been followed for a median time from diagnosis of 14 mo (range 1 to 56 mo). Using standard tumor mass staging, $20 \%$ of patients had a low tumor mass, $32 \%$ had an intermediate, and $48 \%$ had 
a high tumor mass stage (Table I) (1). Treatment consisted of intermittent courses of vincristine, cyclophosphamide, adriamycin, and prednisone, according to standard treatment protocols (12). Few patients received low dose cytosine arabinoside during maintenance phase. Tumor response was defined as $\geq 75 \%$ reduction in serum $M$-protein concentration and/or disappearance of Bence Jones proteinuria. Of 72 evaluable patients, 43 responded $(61 \%)$ and 29 were resistant. The unevaluable patients included two individuals who were lost to follow-up and three patients who died of sepsis within 4 wk of treatment before any antitumor effect could be defined.

In addition, we have also evaluated 29 patients with refractory myeloma, who were treated with a combination of vinca alkaloids, adriamycin, and high dose prednisone (13).

In all patients, marrow aspirates from the posterior iliac crest were obtained under local anesthesia. Smears were prepared for microscopic analysis of the proportion of plasma cells. A separate marrow sample was obtained in a heparinized syringe for flow cytometric $(\mathrm{FCM})^{1}$ analysis. Cells were then subjected to Hypaque-Ficoll density gradient separation and, in the case of DNA content analysis, fixed with ethanol and stained with ethidium bromide and mithramycin (14). In 34 patients, we performed FCM analysis of both DNA and RNA content. This involved staining of unfixed cells with acridine orange, according to the twostep procedure reported by Traganos et al. (15). FCM analysis was performed with an ICP flow cytometer (Phywe, Gottingen, FRG) using filter combinations described previously (16).

The following parameters were derived from FCM analysis of ethidium bromide/mithramycin-stained cells: DNA index (DI), as a measure of ploidy using relative fluorescence intensities of tumor $\mathrm{G}_{1 / 0}$ cells vs. granulocyte controls; and the proportion of $\mathrm{G}_{1 / 0}$ cells with abnormal DNA content (9). FCM analysis of acridine orange-stained cells yielded the following information: RNA index (RI); the ratio of mean RNA content of tumor $G_{1 / 0}$ cells to that of lymphocyte controls (5); and the proportion of $\mathrm{G}_{1 / 0}$ cells with abnormal RNA and/or DNA content. These determinations were accomplished with the use of a "boxogram" gating procedure (10).

Statistical analyses included the following: comparisons of response rates among groups using chi-square tests $(17)$; tests for linear trend in response rates (16); various standard tests of correlation and regression; and a modified Wilcoxon test for differences in survival distributions (18).

\section{RESULTS}

Cytometry-derived measurements and marrow plasmacytosis. Representative examples of bone marrow DNA-RNA histograms of a case each of hyperdiploid and diploid myeloma are depicted in Fig. 1. As verified by in vitro mixing experiments with peripheral blood from normal donors (data not shown; 9,10 ), myeloma tumor cells, in both instances, are characterized by a significantly higher RNA content than residual normal marrow cells. The discrimination between tumor and normal cells is further enhanced

\footnotetext{
${ }^{1}$ Abbreviations used in this paper: DI, DNA index; FCM analysis, flow cytometric analysis; RI, RNA index; RRI, relative $\mathbf{R I}$.
}

TABLE I

Patient Characteristics

\begin{tabular}{lc}
\hline \multicolumn{1}{c}{ Parameter } & No. of patients \\
\hline Immunoglobulin type & \\
IgG & 47 \\
IgA & 14 \\
Light chains only & 14 \\
Nonsecretory & 2 \\
Tumor mass & \\
Low & 15 \\
Intermediate & 25 \\
High & 37 \\
Response & \\
Evaluable & 72 \\
Response & 43 \\
Resistance & 29 \\
Early death & 3 \\
Lost to follow-up & 2 \\
Total & 77 \\
\hline
\end{tabular}

in the case of an additional abnormality in DNA content (Fig. $1 \mathrm{~B}$ ).

Abnormalities in DNA content were present in $79 \%$ of 77 patients, with a median DI of 1.1 (range 0.9 to 1.9). A distinctly elevated RNA content was noted in all 34 patients in whom acridine orange measurements were performed. The median RI was 4.9 (range 1.1 to 17.3). The detection rate by DNA analysis in this group was $80 \%$ and hence similar to our experience in the larger patient sample. Thus, analysis of RNA content using acridine orange in this study population enhances the cytometric detection rate of myeloma tumor cells to $100 \%$. There was a linear correlation between the degree of marrow plasmacytosis and the proportion of tumor cells in the marrow as identified by either cytometric technique (Fig. $2 ; r=0.75, P$ $<0.001$ ).

Marrow tumor infiltrate and clinical tumor mass stage. Fig. 3 depicts a comparison between the proportions of microscopically and cytometrically defined marrow tumor cells as a function of clinical tumor mass stage. With increasing tumor mass, there was evidence of a linear increase in the proportion of both microscopically recognized plasma cells $(P=0.01)$ and of cytometrically abnormal cells, both as measured by DNA $(P<0.01)$ or DNA and RNA $(P<0.01)$.

$R I$, marrow tumor infiltrate, and $M$-protein concentration. For the subgroup of 34 patients with available DNA/RNA FCM measurements, we analyzed the relationship between marrow tumor infiltrate and RI. Excluding two patients with exceptionally high $R I$ values, $>12$, an inverse relationship be- 


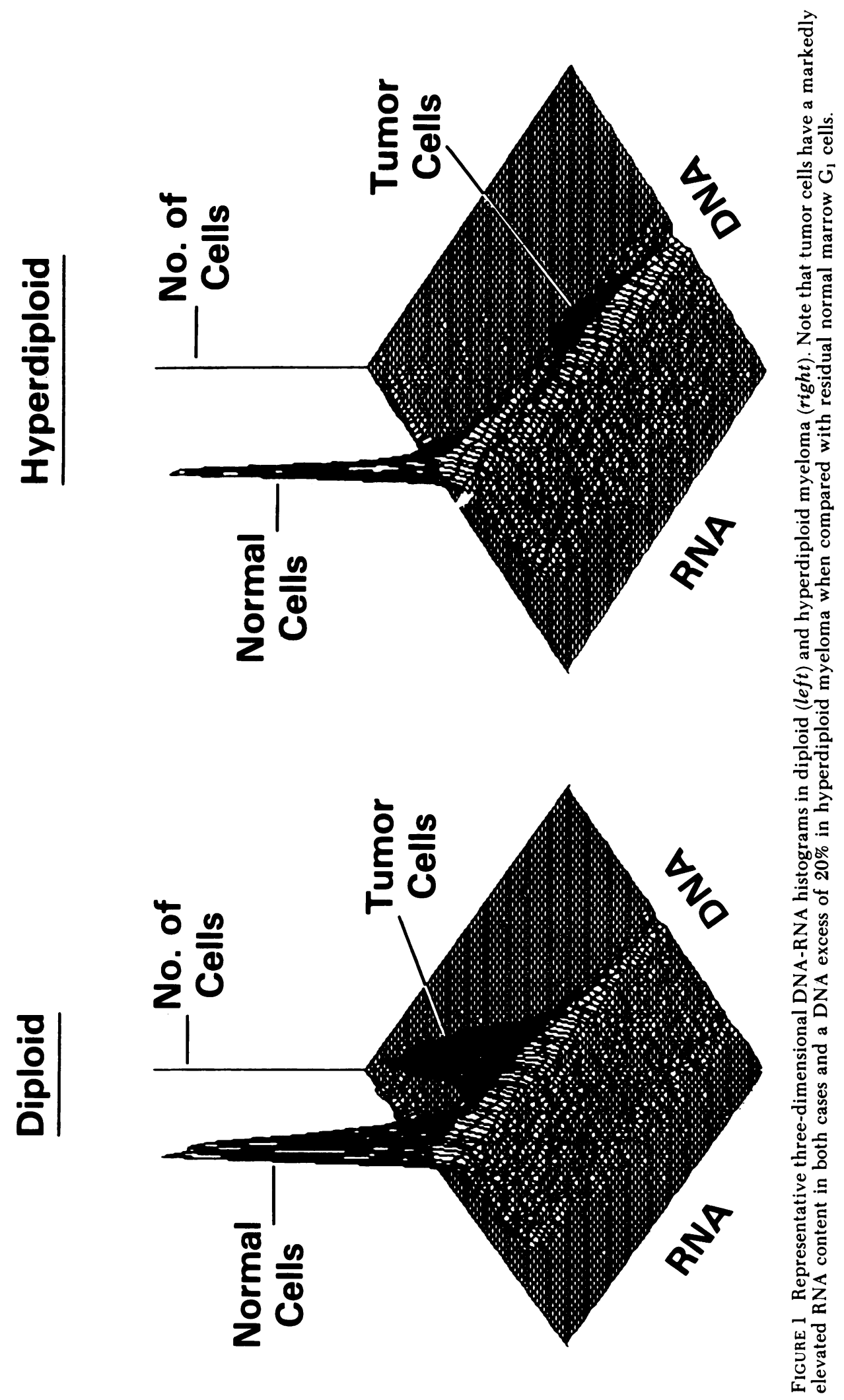




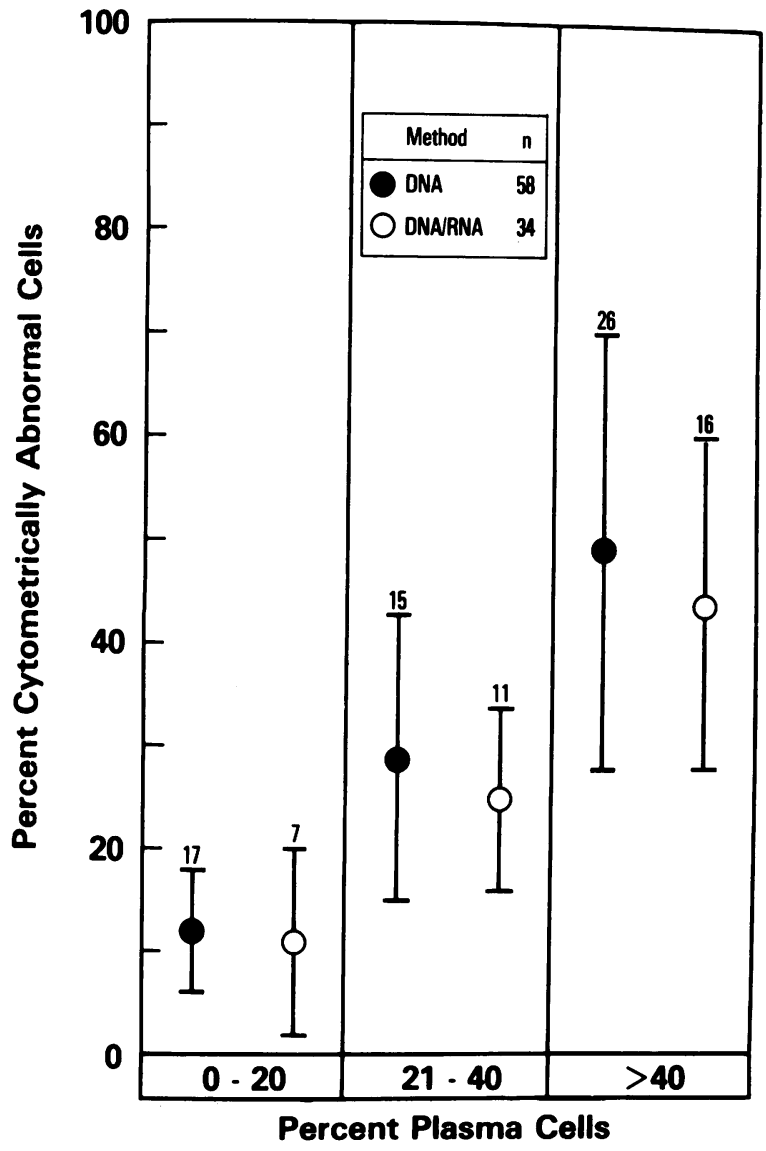

Figure 2 Relationship between morphologic and cytometric assessment of marrow tumor infiltrate in myeloma. Note the linear increment in the proportions of both DNAabnormal cells $(r=0.74 ; P<0.01)$ and DNA/RNA-abnormal cells $(r=0.75 ; P<0.01)$ with increasing degree of marrow plasmacytosis. The data are presented as means \pm SD.

tween these two parameters was noted, i.e., RI decreased with increasing marrow tumor infiltrate $(r$ $=-0.24 ; P=0.08$ ).

Fig. 4 examines the product of RI and the percentage of cytometrically identifiable tumor cells (as a measure of the capacity for protein synthesis) in relationship to serum M-protein concentration. For 16 of 26 patients, there was strong evidence that this product increased linearly with serum M-protein concentration $(r=0.6 ; P=0.01)$. There were 10 individuals including 1 patient with nonsecretory disease in whom, for a given $\mathrm{M}$-protein concentration, the cellular metabolic capacity was considerably higher than in the previous group, suggesting ineffective immunoglobulin secretion. 6 of these 10 patients had a high tumor mass stage compared with 6 of 16 in the aforementioned group.
Prediction of response. Analysis of initial treatment response by parameters related to tumor burden (clinical tumor mass stage morphologic and cytometric assessment of marrow tumor infiltrate) revealed only a weak adverse impact of high clinical tumor mass stage and high degree of marrow plasmacytosis on response rate, which was only apparent in the larger group of 77 patients (Table II). Ploidy, as expressed by the DI, did not systematically affect remission induction, although a low-degree hyperdiploid DI (1.011.15) was associated with the highest and hypodiploidy with the lowest response rate. The strongest single predictor for remission induction was the RI of tumor cells: values $<4$ identified high risk patients with only one of eight responses (13\%), whereas values $>6$ defined a particularly favorable response category $(89 \%$; $P=0.004)$. Joint consideration of RI and marrow tumor infiltrate was accomplished by defining a relative RI (RRI), representing the ratio of these two variables (RI: tumor cell proportion). 93\% of patients with RRI $>20$ achieved remission, contrasting with only $29 \%$ for patients with RRI $\leq 20$ (Fig. 5; $P<0.001$ ).

A similar association between tumor cell RI and response was noted in 29 patients receiving salvage treatment (Table III). The 13 patients with RI > 6 had a

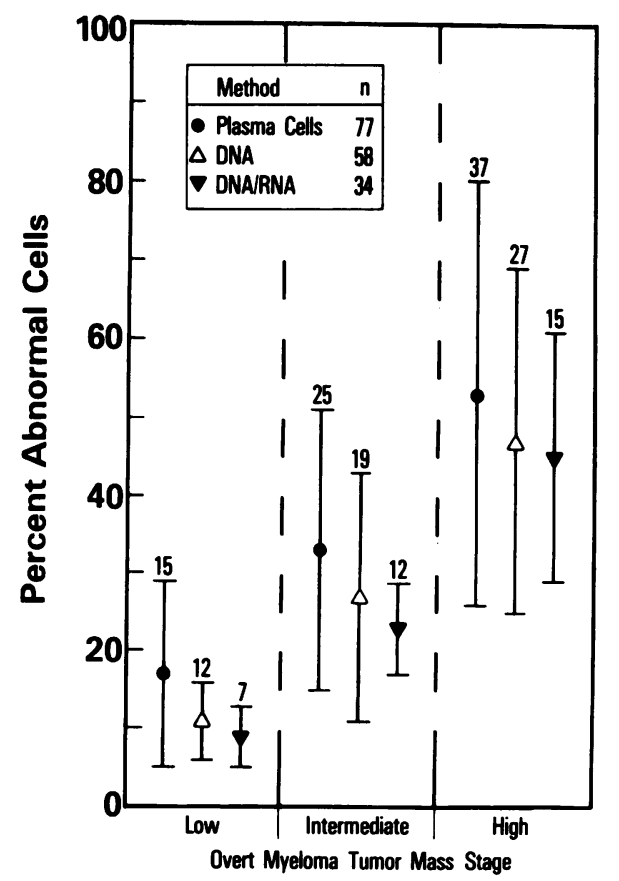

Figure 3 Relationship between marrow tumor infiltrate and clinical estimation of tumor mass stage in myeloma. There is a progressive increment in the marrow tumor infiltrate with increasing tumor mass stage, regardless of the marrow tumor parameter used $(P<0.01)$. 


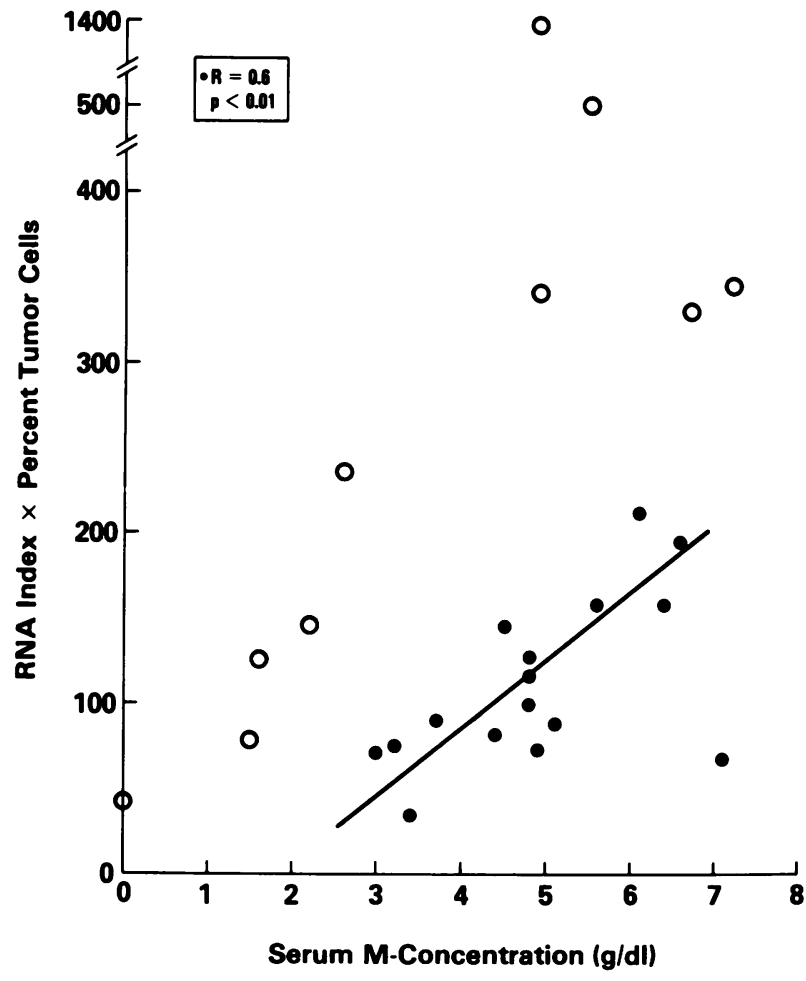

Figure 4 Relationship between tumor cell metabolic capacity, represented by the product of RI and proportion of marrow tumor cells, and serum M-protein concentration. For a subpopulation of 16 patients $(0)$, a linear correlation between these two parameters is noted $(r=0.6 ; P<0.01)$. There were 10 additional individuals (including one patient with nonsecretory myeloma) with disproportionately high cellular metabolic capacity for a given serum M-protein concentration, suggesting ineffective immunoglobulin secretion and/or premature catabolism $(\mathrm{O})$.

significantly higher response rate than patients with low and intermediate values (85 vs. $25 \%, P=0.01$ ).

Prediction of survival. Table IV gives the estimated percentages of patients surviving 1 and $2 \mathrm{yr}$ according to the patient characteristics under investigation. Again, as in Table II, analysis is provided both for those patients in whom all parameters were known and separately for the largest number of patients with any parameter. The patient features most strongly related to survival were tumor mass stage (patients with high tumor mass having poor survival), marrow plasmacytosis, and percentage of cells with abnormal DNA/RNA content (patients with values $>30 \%$ having poor survival) and RRI (patients with values $>20$ having longer survival) (all $P$ values $<0.05$ ). Patients with hypodiploid and diploid myeloma showed a trend towards shorter survival compared with patients with hyperdiploid disease, which was only apparent in the larger patient population $(P=0.12)$.
Fig. 6 illustrates Kaplan-Meier survival plots as a function of tumor mass stage (a), marrow tumor infiltrate by plasmacytosis $(b)$ and DNA-RNA FCM analysis $(c)$, RI $(d)$, and RRI $(e)$. Discrimination of different risk groups was apparent only when tumor mass-related parameters $(a, b, c)$ were considered. As indicated in Table IV, clinical tumor mass stage and degree of marrow plasmacytosis were predictors of survival in both the larger group of 77 patients and in the subset of 34 patients with available DNA-RNA data, indicating that the latter group is a representative one. The tumor cell intrinsic RI parameter, shown to predict for initial treatment response (Table II), did not affect survival, when analyzed in the specified two evenly distributed subgroups with values below and above 5 (Fig. $6 d$ ). However, the derived RRI parameter separated, as did FCM-defined marrow tumor infiltrate $(c)$, good and poor survival risk groups (Fig. 6 $e, P=0.004)$.

TABLE II

Summary of Response by Pretreatment Tumor Mass and FCM Variables

\begin{tabular}{cccc}
\hline Characteristic & $\begin{array}{c}\text { No. of } \\
\text { patients }\end{array}$ & $\begin{array}{c}\text { Percent } \\
\text { responding }\end{array}$ & $P$ \\
\hline
\end{tabular}

Tumor mass

Low

Intermediate

$7(15)^{\circ} \quad 86(80)$

High

$10(20) \quad 60(70)$

$0.22(0.07)$

15 (36) $\quad 47(47)$

DI

$<1.0 \quad 4(6) \quad 25(33)$

1.0

5 (15) $60(47)$

1.01-1.15

$10(23) \quad 70(70)$

$>1.15$

$13(28) \quad 62(64)$

$0.52(0.26)$

Percent plasma cells

$\begin{array}{lrr}\leq 20 & 7(21) & 71(76) \\ 21-45 & 11(21) & 55(62) \\ >45 & 14(30) & 57(47)\end{array}$

$0.76(0.10)$

Percent of DNA/RNA FCM abnormal cells

$\begin{array}{lcc}<20 & 10(19) 1 & 80(68) \\ 20-40 & 14(17) & 50(59) \\ >40 & 8(18) & 50(56)\end{array}$

$0.28(0.7)$

RI $\begin{array}{lrr}<4 & 8 & 13\end{array}$

$\begin{array}{lll}4-6 & 15 & 67\end{array}$

$\begin{array}{lll}>6 & 9 & 89\end{array}$

0.004

RRI

$\begin{array}{llll}\leq 20 & 17 & 29 & <0.001 \\ >20 & 15 & 93 & \end{array}$

( $)^{\circ}$ all patients with this particular characteristic.

( ) 1 patients with DNA FCM abnormal cells, i.e., excluding diploid disease. 


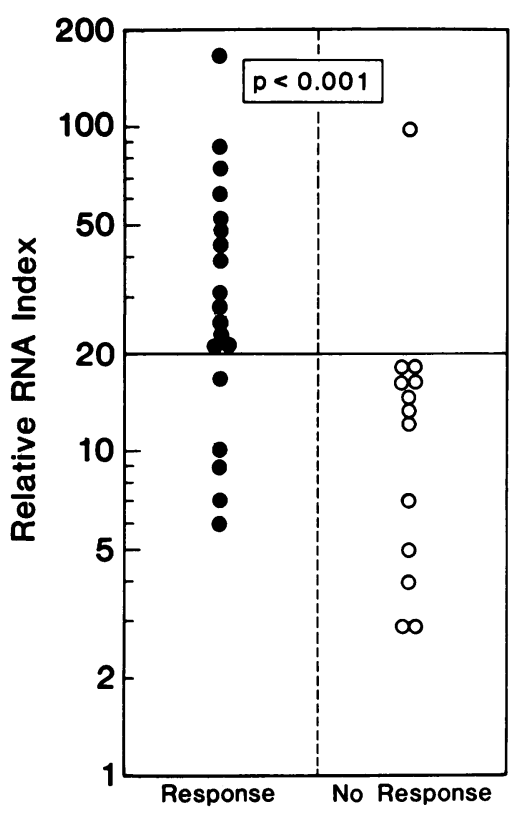

Figure 5 RRI in myeloma. Response discrimination in previously untreated patients with myeloma according to RRI. Patients with RRI values $>20$ have a $93 \%$ response rate compared with $29 \%$ for individuals with $\leq 20(P<0.001)$.

\section{DISCUSSION}

The present investigation confirms our earlier reports on the usefulness of FCM analysis to objectively enumerate myeloma tumor cells in heterogeneous bone marrow $(5,9,10)$. In addition, we demonstrate biological and clinical implications of bone marrow tumor infiltrate using both standard morphologic and cytometric means. Thus, the proportional increase in marrow tumor infiltrate with increasing tumor mass stage indicates the usefulness of the widely neglected plasma cell differential for the direct definition of tumor mass grade. This was further supported by the prognostic association also of plasmacytosis with survival. Analysis of the difference between marrow plasmacytosis and cytometry-derived measurements of marrow tumor infiltrate revealed that for $85 \%$ of all patients, the dif-

TABLE III

RI and Response in Previously Treated Myeloma

\begin{tabular}{cccc}
\hline RI & No. of patients & Percent responding & $P$ \\
\hline$<4$ & 4 & 25 & \\
$4-6$ & 12 & 25 & $<0.01$ \\
$>6$ & 13 & 83 & \\
Total & 29 & 52 & \\
\hline
\end{tabular}

ference between these two measurements did not exceed $20 \%$. The highest incidence of $>20 \%$ discrepancy was observed in individuals with high tumor mass stage (25\%), while there was complete conformity for patients with low tumor mass. There was no difference in either response rate or survival for patients with concordant or discordant morphologic vs. cytometric marrow tumor infiltrate measurements. The relatively low incidence (15\%) of marked discrepancy $>20 \%$ between microscopic and cytometric examinations stresses that patchiness of marrow involvement by myeloma is the exception, as is solitary osseous myeloma (19). The former conclusion was further substantiated by comparison between cytometry-derived measurements of marrow infiltrate in simultaneously obtained aspirates and biopsies from 15 patients, where a correlation coefficient $r=0.89$ was found $(P$ $<0.01)$. Surprisingly, in six of seven cases with $>10 \%$ difference $(<15 \%)$ in tumor infiltrate between the two tissue sources, marrow aspirates contained the larger proportion of tumor cells. Thus, while not providing the same objectivity and reproducibility as the cytometric differential, the degree of marrow plasmacytosis by microscopic examination may be a useful ancillary tool for staging of myeloma in those institutions lacking cytometry facilities. This is particularly true for clinical situations where the laboratory parameters used for myeloma staging may be altered by nonneoplastic conditions affecting hemoglobin and renal function tests. Our observation of an approximately 1.5to twofold higher marrow infiltrate in intermediate and three to four times higher infiltrate in high tumor mass disease than in patients with low tumor mass conforms well with previous observations of Durie et al. (1) using information on in vitro immunoglobulin production rates.

The trend of decreasing RI with increasing marrow tumor infiltrate suggests that myeloma with low RI may be a more aggressive disease, presenting with a more advanced tumor mass stage when first diagnosed. The alternative interpretation of decreasing RI with tumor expansion in the same patient is unlikely in view of stable RI measurements in patients studied serially. High RI and low marrow tumor infiltrate are consistent with a more differentiated and earlier stage myeloma including indolent disease, ${ }^{2}$ which is associated with a greater sensitivity to chemotherapy (see below).

Of interest was the correlation between immunoglobulin production capacity, as assessed by the product of RI and marrow tumor infiltrate, and serum Mprotein concentration. There was a subgroup of

\footnotetext{
${ }^{2}$ Barlogie, B. Unpublished observations.
} 
TABLE IV

Summary of Survival by Pretreatment Tumor Mass and FCM Variables

\begin{tabular}{|c|c|c|c|c|c|c|}
\hline \multirow[b]{2}{*}{ Characteristic } & \multirow[b]{2}{*}{ No. of patients } & \multirow[b]{2}{*}{ No. dead } & \multicolumn{2}{|c|}{ Percent surviving } & \multirow{2}{*}{\multicolumn{2}{|c|}{$P$}} \\
\hline & & & $1 \mathrm{yr}$ & $2 \mathrm{yr}$ & & \\
\hline \multicolumn{7}{|l|}{ Tumor mass } \\
\hline Low/intermediate & $19(40)^{\bullet}$ & $6(19)$ & $84(91)$ & $51(67)$ & \multirow{2}{*}{0.07} & \\
\hline High & $15(37)$ & $9(27)$ & $52(61)$ & $11(32)$ & & $(0.002)$ \\
\hline \multicolumn{7}{|l|}{ Percent plasma cells } \\
\hline$\leq 30$ & $15(35)$ & $3(17)$ & $87(85)$ & $77(66)$ & \multirow{2}{*}{0.04} & \multirow{2}{*}{$(0.05)$} \\
\hline$>30$ & $19(42)$ & $12(29)$ & $58(73)$ & $13(37)$ & & \\
\hline \multicolumn{7}{|l|}{ DI } \\
\hline$\leq 1$ & $10(22)$ & $5(17)$ & $63(67)$ & $42(42)$ & \multirow{2}{*}{0.8} & \multirow{2}{*}{$(0.12)$} \\
\hline$>1$ & $24(55)$ & $10(29)$ & $77(83)$ & $23(55)$ & & \\
\hline \multicolumn{7}{|c|}{$\begin{array}{l}\text { Percent DNA/RNA FCM } \\
\text { abnormal cells }\end{array}$} \\
\hline$\leq 30$ & $17(27) \ddagger$ & $5(13)$ & $94(85)$ & $45(61)$ & \multirow{2}{*}{\multicolumn{2}{|c|}{$0.004(0.1)$}} \\
\hline$>30$ & $17(31)$ & $10(19)$ & $47(77)$ & $24(41)$ & & \\
\hline \multicolumn{7}{|l|}{ RI } \\
\hline$\leq 5$ & 18 & 9 & 73 & 45 & \multirow{2}{*}{0.8} & \\
\hline$>5$ & 16 & 6 & 72 & 31 & & \\
\hline \multicolumn{7}{|l|}{ RRI } \\
\hline$>20$ & 15 & 2 & 92 & 59 & \multirow{2}{*}{0.004} & \\
\hline$\leq 20$ & 19 & 13 & 54 & 28 & & \\
\hline
\end{tabular}

patients who displayed, for a given M-protein concentration, an inappropriately high tumor metabolic capacity, the extreme being represented by patients with nonsecretory myeloma (20). Such ineffective immunoglobulin production is consistent with the occurrence of premature immunoglobulin catabolism both intracellularly and extracellularly in some patients.

One of our salient observations consists of the prognostic implications of the cellular features of myeloma derived from one single marrow examination. Thus, tumor cell RI and RRI were associated with remission induction, whereas the cytometrically defined marrow tumor infiltrate and RRI, but not RI by itself, correlated with survival. Hence, although the same derived FCM parameter (RRI) affected both short- and longterm prognosis, the tumor cell intrinsic RI seemed to impact on remission induction (in both untreated and refractory myeloma), and the tumor burden-related marrow tumor infiltrate predominantly determined survival. The present study group is too small to assess whether the survival implications of the FCM analysisderived pretreatment variables apply both in responding patients and in the treatment failure group. The greater sensitivity to chemotherapy of myeloma pre- senting with high RI (suggesting a higher degree of tumor differentiation) and low tumor mass is poorly understood. We do not have evidence for a cytokinetic mechanism, such as higher growth fraction in the presence of low tumor burden, favoring chemotherapy sensitivity. In fact, data by Hofmann et al. (8) on thymidine grain count would suggest that myeloma with low DNA synthetic activity is more responsive to induction chemotherapy. This observation is compatible with our results, if one assumes that more differentiated myeloma with high RI is characterized by lower proliferative activity. A superior chemotherapy response in low compared with high proliferative activity tumors does not fit conventional concepts of cell kinetics. The higher response rate in high RI and low tumor burden myeloma may reflect a smaller proportion of drug-resistant cell subpopulations as recently suggested by Goldie et al. (21). Research is ongoing in our laboratory to determine the interrelationship between tumor cell RI, cytokinetics, and tumor mass stage, in an effort to further define the predictive power of pretreatment cellular measurements. The development of prognostic models for incidence and duration of remission and for survival should enable 

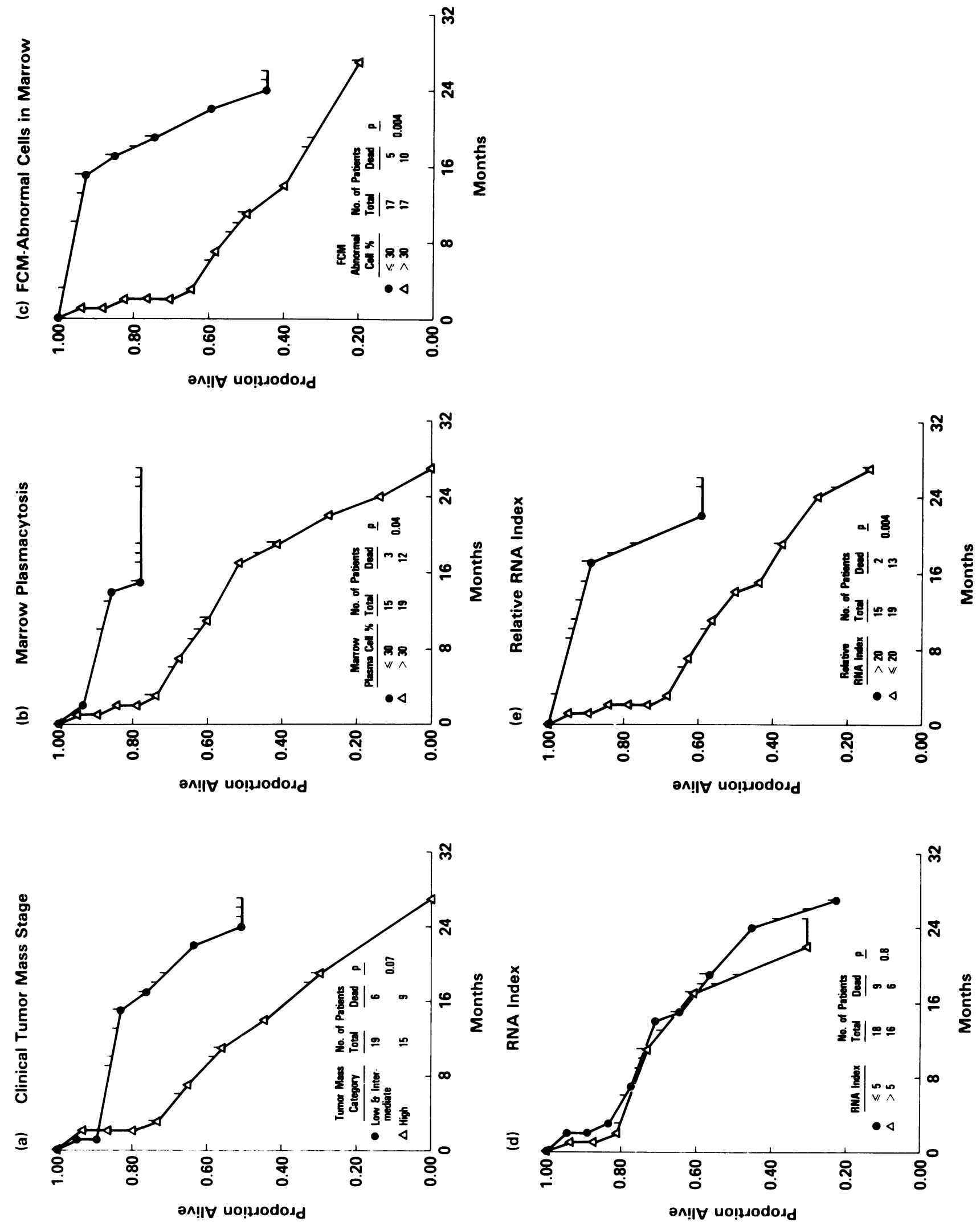
us to investigate new agents in high risk patients during various phases of their disease, as has been successfully done in adult acute leukemia $(22,23)$.

\section{ACKNOWLEDGMENTS}

The authors wish to express their appreciation to Mattie J. Thomas for her excellent secretarial assistance.

This work was supported, in part, by grant CA28771 from the National Institutes of Health, National Cancer Institute.

\section{REFERENCES}

1. Durie, B. G. M., and S. E. Salmon. A clinical staging system for multiple myeloma. Cancer. 36:842-854.

2. Alexanian, R., S. Balcerzak, J. Bonnet, E. Gehan, A. Haut, J. Hewlett, and R. Monto. 1975. Prognostic factors in multiple myeloma. Cancer. 36:1192-1201.

3. Bergsagel, D. E. 1975. Plasma cell meyloma: prognostic factors and criteria of response to therapy. In Cancer Therapy. M. J. Staquet, editor. Raven Press, New York. 78-87.

4. Durie, B., S. Salmon, and T. Moon. 1980. Pretreatment tumor mass, cell kinetics, and prognosis in multiple myeloma. Blood. 55:364-372.

5. Latreille, J., B. Barlogie, D. Johnston, B. Drewinko, and R. Alexanian. 1982. Ploidy and proliferative characteristics in monoclonal gammopathies. Blood. 59:43-51.

6. Alexanian, R., S. Salmon, J. Gutterman, D. Dixon, J. Bonnet, and A. Haut. 1981. Chemoimmunotherapy for multiple myeloma. Cancer. 47:1923-1929.

7. Medical Research Council. 1980. Treatment comparisons in the third MRC myelomatosis trial. Br. J. Cancer. 42:823-830.

8. Hofmann, W., S. Salmon, and B. G. M. Durie. 1981. Drug resistance in multiple myeloma associated with high in vitro incorporation of ${ }^{3} \mathrm{H}$-thymidine. Blood. $58: 471-476$.

9. Latreille, J., B. Barlogie, G. Dosik, D. Johnston, B. Drewinko, and R. Alexanian. 1980. Cellular DNA content as a marker of human multiple myeloma. Blood. 55:403408.

10. Barlogie, B., J. Latreille, D. Swartzendruber, R. Alexanian, L. Smallwood, A. M. Maddox, M. Raber, and B. Drewinko. 1982. Quantitative cytology in myeloma research. In Clinics in Haematology. William R. Schmidt, editor. W. B. Saunders Co., New York. 19-45.
11. Alexanian, R. 1980. Localized indolent multiple myeloma. Blood. 56:521-526.

12. Durie, B. G. M., and S. Salmon. 1982. The current status and future prospects of treatment for multiple myeloma. In Clinics in Haematology. William R. Schmidt, editor. W. B. Saunders Co., New York. 181-210.

13. Alexanian, R., B. S. Yap, and G. Bodey. 1983. Prednisone pulse therapy for refractory myeloma. Blood. In press.

14. Barlogie, B., G. Spitzer, J. Hart, D. Johnston, T. Buchner, J. Schumann, and B. Drewinko. 1976. DNA histogram analysis of human hemopoietic cells. Blood. 48:245-257.

15. Traganos, F., Z. Darzynkiewicz, T. Sharpless, and M. R. Melamed. 1977. Simultaneous staining of ribonucleic and deoxyribonucleic acids in unfixed cells using acridine orange in a flow cytofluorometric system. J. Histochem. Cytochem. 35:46-53.

16. Barlogie, B., J. Latreille, E. J. Freireich, C.-T. Fu, D. Mellard, M. Meistrich, and M. Andreeff. 1980. Characterization of hematologic malignancies by flow cytometry. Blood Cells. 5:719-744.

17. Cochran, W. G. 1954. Some methods for strengthening the common $\chi^{2}$ tests. Biometrics. 10:417-451.

18. Gehan, E. 1965. A generalized Wilcoxon test for comparing arbitrarily singly censored samples. Biometrika. 52:203-224.

19. Bataille, R. 1982. Localized plasmacytomas. In Clinics in Haematology. William R. Schmidt, editor. W. B. Saunders Co., New York. 113-122.

20. Preud'Homme, J. L., S. Labaume, and W. Praloran. 1980. Synthesis of abnormal heavy chains in Bence Jones plasma cell leukemia with intracellular IgG. Blood. 56:1136-1140.

21. Goldie, J. H., and A. J. Coldman. 1979. A mathematical model for relating the drug sensitivity of tumors to their spontaneous mutation rate. Cancer Treat. Rep. 63:17271733.

22. Freireich, E. J., E. A. Gehan, J. F. Speer, L. Heilbrun, T. Smith, G. P. Bodey, K. B. McCredie, V. Rodriguez, J. S. Hart, and M. A. Burgess. 1973. The usefulness of multiple pretreatment patient characteristics for prediction of response and survival in patients with adult acute leukemia. In Advances in the Biosciences. Workshop on Prognostic Factors in Human Acute Leukemia. T. M. Fliedner and S. Perry, editors. Pergamon Press, New York. 14:131-144.

23. Keating, M. J., T. L. Smith, E. A. Gehan, K. McCredie, G. P. Bodey, G. Spitzer, E. Hersh, J. Gutterman, and E. J. Freireich. 1980. Factors related to length of complete remission in adult acute leukemia. Cancer. 45:27792029.

FIGURE 6 Survival in myeloma by tumor mass-related parameters and by tumor intrinsic cellular properties. (a) Significantly shorter survival in high vs. intermediate and low tumor mass stage (median of 14 vs. 35 mo, $P=0.002$ ). (b) Shorter survival in myeloma with $>30 \%$ vs. $\leq 30 \%$ marrow plasmacytosis (median of 20 vs. $40 \mathrm{mo}, P=0.05$ ). (c) Adverse effect of cytometrically defined marrow tumor infiltrate $>30 \%$ on survival (median of $22 \mathrm{vs.} 11 \mathrm{mo}$, $P=0.004)$. (d) No difference in length of survival for patients with high $(>5)$ vs. low RI $(\leq 5)$ $(P=0.8)$. (e) Ratio of RI and marrow tumor infiltrate (RRI), already noted to predict for remission induction (Table II and Fig. 5), provides excellent separation into good and poor risk groups; high values $(>20)$ identifying favorable group $(26+$ vs. $14 \mathrm{mo}, P=0.004)$. 